\title{
Factores de riesgo psicosocial predictores del Síndrome de Quemarse por el Trabajo (SOT) (Burnout) en docentes de Bachillerato
}

\section{Psychosocial Risk Factors predictors of Burnout in High School teachers.}

Fecha de recepción: 6-05-2019

Fecha de aceptación: 31-01-2020

\begin{abstract}
Sara Unda Rojas
Universidad Nacional Autónoma de México. Facultad de Estudios

Superiores Zaragoza.

Rosa Alejandra Hernández-Toledano

Universidad Nacional Autónoma de México. Facultad de Estudios

Iztacala. México.

Oscar García-Arreola

Universidad Nacional Autónoma de México. Facultad de Estudios

Iztacala. México

Carlos Esquivel Lozada

Universidad Nacional Autónoma de México. Facultad de Estudios
\end{abstract}

Superiores Zaragoza.

\section{resumen/alostract:}

El objetivo fue construir un modelo predictivo del SQT en profesores a nivel Bachillerato. Se utilizó un diseño correlacional, transversal con muestreo no probabilístico. Participaron 395 Docentes de Bachillerato. La prevalencia del SQT fue de 17.6\%. El estresor con mayor prevalencia fue Estudiantes difíciles (ED) seguido por una Mala Relación con Compañeros (MRC). Los predictores de la Baja Ilusión fueron: Falta de recursos para realizar su trabajo y Falta de apoyo del jefe (FAJ) además de Estudiantes Difíciles; para el Desgaste psíquico (DP): los predictores fueron MRC, Sobrecarga académica (SA) e Inequidad. Para Indolencia (I): Ios ED, FAJ y SA para la Culpa: fue la Indolencia. Los resultados del modelo fueron $X^{2}=49.88, \mathrm{gl}=1 X^{2} / \mathrm{gl}=3 p<.05 ; \mathrm{IFl}=.976, \mathrm{TLI}=.930, \mathrm{CFI}=.976, \mathrm{GFI}=.976$ y un $\mathrm{AGFl}=$ $.916, \mathrm{RMSEA}=.073$. En conclusión, existen factores de riesgo psicosocial en el trabajo dentro del ámbito docente que anteceden al SQT.

The aim was to build a predictive model of Burnout in Senior High-School teachers. A cross-sectional method was used, ex post facto, correlational field, with a non-intentional sampling. 395 high school teachers participated. Prevalence of Burnout was of $17.6 \%$. The highest stressor prevalence was Difficult students (DS) followed by Bad relationship with partners (BRP). The structural model considered predictors of Loss enthusiasm toward the job: Lack of resources and Lack of support from the boss (LSB) and DS for Psychological exhaustion (PE): The BRP; the academic overload (AO) and Inequality; for indolence DS, LSB and $A O$. Guilt is predicted by Indolence. At the path analysis the goodness of fit indexes was: $X^{2}=49.88, d f .=1 X^{2} / d f .=3 p<.05 ; \mid F I=.976, T L I=.930, C F I=.976, G F I=.976$ and $A G F I=.916, R M S E A=.073$. In conclusion there are specific psychosocial risk factors in teaching that predict Burnout.

\section{palabras clave/keywords:}

Estrés laboral; Condiciones de trabajo; Educación media superior; Modelo predictivo.

Job Stress; Working conditionss; Senior high school; Predictive model 


\section{Introducción}

El mundo del trabajo tiene altas exigencias y efectos sobre los trabajadores de todos los niveles, incluyendo a profesionistas. A nivel global se observan impactos desfavorables en las esferas económica, política, social, cultural y de salud de la población trabajadora (Sánchez de Gallardo y Mantilla de Gil, 2005).

\section{Factores de Riesgo Psicosocial en el trabajo.}

Los factores psicosociales en el trabajo definidos como las condiciones presentes en una situación laboral directamente relacionadas con: la organización del mismo, el contenido del puesto, la realización de la tarea o incluso con el entorno de trabajo, son capaces de afectar positiva o negativamente el desarrollo del trabajo así como la salud del trabajador. Cuando éstos factores actúan de forma negativa en el trabajador se convierten en "factores de riesgo psicosocial en el trabajo" (FRPS-T); producirán tensión en el individuo impactando además negativamente en la productividad y sobre todo en la salud-

Los FRPS-T capaces de generar estrés han sido documentados por diversos autores, algunos señalan la introducción de nuevas tecnologías, organización de clases, preparación de informes, elaboración de estadísticas, reuniones excesivas, falta de reconocimiento profesional de los jefes, número excesivo de horas, exceso de responsabilidades, desinterés, indisciplina y actitudes desafiantes de los alumnos, espacio físico, suciedad en el mobiliario, falta de iluminación, turnos mixtos, horarios, excesivas, diversas tareas, poca estabilidad en el empleo, carencia de material didáctico, inseguridad intralaboral (casos como robos y asaltos), injusticia e inequidad en el trato y la contratación, así como en el acceso a recursos, burocracia, conflictos con compañeros, favoritismo, falta de recursos, simulación, autoritarismo y discriminación (Caballero, 2018; López y Gil-Monte, 2015 y Unda, 2016).

Los FRPS-T están relacionados con manifestaciones psicofisiológicas cuando el trabajador percibe situaciones laborales como estresantes, y al llevar a cabo estrategias de afrontamiento fallidas, se abre la posibilidad de abrir paso a consecuencias negativas como el Síndrome de quemarse por el trabajo (SQT) (Ratto, García, Silva y González, 2015).

\section{El síndrome de Quemarse por el Trabajo (Burnout)}

Maslach (2003), considera al Síndrome una respuesta a estresores crónicos a nivel personal y relacional en el trabajo de carácter tridimensional: Agotamiento emocional, Sentimientos de cinismo y Falta de realización profesional. Estas características son evaluadas a través del Maslach Burnout Inventory (MBI), un instrumento ampliamente usado en países de habla inglesa, aunque en países de habla hispana presenta algunos problemas psicométricos particularmente en la escala de despersonalización (Olivares y Gil-Monte, 2009).

Gil-Monte (2005), reconceptualiza las dimensiones mencionadas por Maslach y propone un modelo que integra las dimensiones de Desgaste psíquico, Indolencia y Baja Ilusión por el trabajo respectivamente, pero a diferencia del modelo del MBI, le integra una cuarta dimensión definida como Culpa y define al SQT como la "respuesta al estrés laboral crónico que aparece en los profesionales de organizaciones de servicios que trabajan en contacto con usuarios de la organización" (p. 14). 
El modelo de Gil-Monte (2005) distingue dos perfiles clínicos del SQT de acuerdo a su gravedad. El primero de ellos (Perfil 1) se desarrolla cuando las condiciones de trabajo exponen al profesional a un estrés crónico mermando de una manera considerable su Ilusión por el trabajo y manifestando sentimientos de Desgaste psíquico de forma concomitante, en un segundo momento el profesional presenta actitudes de Indolencia como estrategia de afrontamiento ante las exigencias laborales. En esta etapa el comportamiento del profesional es disfuncional en el trabajo, aún así, las actitudes negativas le evitan sucumbir, pero al no cambiar la situación laboral estos síntomas alcanzan altos niveles, y deviene el síndrome.

Pero eso no es todo, acorde a este modelo, cuando las tres dimensiones se combinan con sentimientos de Culpa, que surgen cuando el profesional se incomoda con sus propias actitudes indolentes y trata de recobrar la motivación acrecentando nuevamente su dedicación e Ilusión, se repite el círculo con manifestaciones peores de Desgaste psíquico y acrecentando el trato indolente que proporciona a los clientes, hasta no poder más, lo anterior permite identificar el perfil 2 de SQT, el más grave, que se acompaña de daños a la salud y de deseos enormes de abandonar el trabajo.

Estos factores se evalúan con el Cuestionario de Evaluación del Síndrome de Quemarse por el Trabajo (CESQT) (Gil- Monte, Viotti y Converso, 2017) que incluye los indicadores de: deterioro cognitivo (medido por las bajas puntuaciones en Ilusión por el trabajo), deterioro afectivo (medido por las altas puntuaciones en Desgaste psíquico y altas puntuaciones en Indolencia) y a la larga pueden presentarse altas puntuaciones en los sentimientos de Culpa (medido por sentimientos de Culpa reportados debido a las actitudes negativas desarrolladas en el trabajo hacia las personas con la que se establecen relaciones laborales).

\section{Factores de Riesgo Psicosocial en el Trabajo y SOT en docentes}

De acuerdo con Blanch (2008) el modelo capitalista y las políticas educativas derivadas del mismo, han impulsado cambios sin precedentes en la profesión docente a nivel mundial. En México los cambios iniciaron en el siglo pasado y se acentúan con la aprobación de la Reforma Educativa impulsada por el grupo de empresarios "mexicanos primero" y a iniciativa del presidente Peña Nieto (Ducoing, 2018). La intención es una escuela que cubra las necesidades de estudiantes-clientes y responda a las necesidades de un mundo globalizado y competitivo en los campos de la innovación tecnológica y la investigación. Dentro del contexto con los cambios laborales se incrementa en el personal docente la prevalencia de problemas de salud mental y de malestar (Rabasa, Figuereido-Ferraz, Gil-Monte y LlorcaPellicer, 2016) además de altos niveles de estrés (García-Carmona, Marín y Aguayo, 2019). Aloe, Amo, y Shanahan (2014) refieren que los profesores son más vulnerables a los efectos del síndrome que otros trabajadores, con hallazgos a nivel mundial que lo confirman (Fernández-Puig, Longás-Mayayo, Chamarro- Lusarby y Virgili-Tejedor 2015; Villaverde, Unda, Escotto-Cordova y Flores 2019).

Trabajos de investigación (Caballero, 2018; López y Gil-Monte, 2015) reportan que cuando un docente se encuentra expuesto a situaciones estresantes crónicas en su trabajo y desarrolla SQT, tiene como consecuencia sentimientos de poca motivación en la ejecución profesional y afecta las relaciones con las personas que son parte de la actividad docente, así 
como la calidad de la enseñanza. A nivel social el síndrome afecta a la misión de la escuela y a la calidad de la enseñanza (Serrano-Díaz y Guil-Bozal, 2014). A nivel organizacional el efecto se observa en la rotación de personal y el ausentismo (Fernet, Guay, Sénecal y Austin, 2012) y a nivel individual, la presencia del SQT se relaciona con la adquisición de hábitos poco saludables como el consumo de alcohol, tabaco, café, drogas, té, estimulantes y tranquilizantes, con amplias repercusiones en la salud mental, física y tiene efectos negativos en el profesional de forma gradual, progresiva y contagiosa (Palacios, Morales y Estrella, 2018).

En cuanto a la prevalencia de SQT en docentes de Colombia muestran porcentajes superiores al 20\% de SQT (Marenco-Escuderos y Ávila-Toscano, 2016), en Portugal, 14.2\% de profesores con niveles elevados de SQT y $1.9 \%$ en niveles críticos (Figueiredo-Ferraz, Gil-Monte y Grau-Alberola, 2009). Fernández-Puig, Longás-Mayayo, Chamarro-Lusarby y Virgili-Tejedor (2015), señalan que los niveles altos se pueden considerar entre el 3 al 13\% en diversas muestras. En Montevideo $21.4 \%$ presentaron niveles altos de SQT (Ratto, García, Silva, González, 2015). En el caso de México, los estudios de aspectos psicosociales asociados al SQT tienen diferencias metodológicas y conceptuales pues no todas abordan el modelo de Maslach y criterios de corte homogéneos (Juárez-García, 2014). El modelo de Gil-Monte es retomado en diversos estudios como el realizado por Unda, García, Hernández, Esquivel y Tovalín (2016) que señalan en una muestra de 939 docentes de la Ciudad de México una prevalencia del SQT de $13.8 \%, 28.5 \%$ con baja Ilusión en el trabajo, 28.2 $\%$ alto Desgaste psíquico, $30 \%$ alta Indolencia, y $23.8 \%$ de alta Culpa. Villaverde, Unda, Escotto-Córdoba y Flores (2019) en una muestra de 375 profesores de Educación básica, señalan que ningún profesor presentó alta Ilusión en el Trabajo, 47.7 \% Desgaste Psíquico en nivel alto o crítico, en cuanto a Indolencia el $39.2 \%$ se presentó nivel alto y crítico, 31.5 $\%$ niveles altos y críticos de Culpa y SQT15 que fue el más alto con un $77 \%$ de los profesores dentro del parámetro alto o crítico.

Por otra parte, Caballero (2018) señala algunas condiciones laborales de los educadores asociadas al síndrome como la falta de apoyo social, relaciones negativas entre los compañeros docentes, la naturaleza compleja del acto de enseñar, como impartir conocimientos, atender alumnos, atender demandas de padres, además de la adaptación a cambios legislativos y pedagógicos. Unda, et al. (2016), señalan como factores de riesgo psicosocial en docentes universitarios a la Inequidad en el trabajo, los Estudiantes difíciles, la Sobrecarga académica, la Falta de recursos y la Inseguridad. Para Fernet, Guay, Sénecal y Austin (2012) una de las principales variables que determinan el SQT es "el ambiente de trabajo dentro de la escuela" (p.522) que incluye aspectos como: Sobrecarga de trabajo, equipo deficiente, las políticas escolares, el clima laboral, los conflictos interpersonales, y los problemas de conducta de los estudiantes.

Estas aproximaciones no rinden cuenta clara de la relación que tienen los factores de riesgo psicosocial con algún síntoma del síndrome, por lo que el objetivo principal de este trabajo es observar si los FRPS-T propios del ámbito docente están correlacionados con las dimensiones del SQT, en caso afirmativo, construir un modelo predictivo entre los FRPS-T y las dimensiones del SQT desde el modelo de Gil-Monte (2005) en docentes de Bachillerato de 
una Universidad Mexicana. Como objetivos secundarios, se consideró establecer la prevalencia tanto del Síndrome como de los FRPS-T de los docentes.

\section{Hipótesis}

H1. Se espera una presencia de SQT y de FRPS-T en esta población de maestros de Bachillerato.

H2. Se espera una asociación de los FRPS-T propios de la labor docente con las dimensiones del síndrome en la población de profesores de Bachillerato.

H3. El modelo de FRPS-T propuesto será un predictor del SQT y sus dimensiones en docentes de Bachillerato.

\section{Material y Métodos}

Participantes: Se realizó muestreo no probabilístico intencional, participaron 395 docentes universitarios de diez centros de dos modalidades de nivel Bachillerato, Colegios de Ciencias y Humanidades (CCH) a saber: plantel Sur 90 (22.8\%), plantel Azcapotzalco 77 (19.5\%) plantel Oriente 73 (18.5\%), plantel Naucalpan 65 (16.5\%), plantel Vallejo, 60 $(15.2 \%)$ y de Escuelas Nacional Preparatoria (ENP) del plantel 224 (6.1\%), plantel 4, 1 profesor $(0.3 \%)$, plantel $6,2(0.5 \%)$, plantel 7,1 profesor $(0.3 \%)$ plantel $9,2(0.5 \%)$ todos pertenecientes a una Universidad Mexicana.

Del total 198 (50.1\%) fueron Hombres y 197 Mujeres (49.9\%). Con una media de edad de $\mathrm{M}=46.58$ años y $\mathrm{DT}=10.57$ (23-75). La antigüedad con una media de $\mathrm{M}=18.69$ años, DT=11.63 (1-50 años). El 63.5\% tiene pareja; 5.3\% tiene estudios de doctorado, 36.5\% maestría y $58.2 \%$ licenciatura. En relación a su nombramiento, el $76.5 \%$ son de Asignatura y $23.5 \%$ de Carrera (Tiempo completo), $54.7 \%$ tiene contrato temporal y $85.8 \%$ está en algún programa de estímulos.

Instrumentos: Se utilizó el Cuestionario de Evaluación del Síndrome de Quemarse por el Trabajo (CESQT) validado en maestros mexicanos por Gil-Monte, Unda y Sandoval (2009). El cuestionario está formado por 20 ítems que se distribuyen en 4 dimensiones: Ilusión por el trabajo (5 ítems) $(\alpha=.76)$, Desgaste psíquico ( 4 ítems) $(\alpha=.82)$, Indolencia (6 ítems, $\alpha=.73$ ) y Culpa (5 ítems, $\alpha=.79)$. Los reactivos se evaluaron con cinco opciones de respuesta tipo Likert que consideraban de cero (nunca) a cuatro (muy frecuentemente: todos los días). La evaluación se realizó conforme al manual del CESQT (Gil-Monte, 2011). El SQT15 se integró con puntuaciones Muy bajas de Ilusión en el trabajo, críticas en Desgaste psíquico, e Indolencia, aquí se consideró el perfil 1. El perfil 2 se integró con los que cumplieron el anterior criterio y adicionalmente puntuaron en nivel crítico en Culpa.

Por otra parte se empleó la Escala de Evaluación de Factores de Riesgo Psicosocial en el Trabajo para maestros universitarios (EFRPS-TU), Unda, et al. (2016), adaptada y validada para docentes de Bachillerato, está compuesta de 26 ítems que se distribuyen en 8 dimensiones: Inequidad ( 4 ítems, $\alpha=.81$ ), Falta de apoyo del jefe ( 3 ítems, $\alpha=.83$ ), Falta de recursos para realizar su trabajo ( 3 ítems, $\alpha=.70$ ), Estudiantes difíciles ( 3 ítems, $\alpha=.83$ ), Mala relación con compañeros ( 3 ítems, $\alpha=.83$ ), Percepción de inseguridad ( 3 ítems,$\alpha=.77$ ), Es- 
tresores físicos ( 4 ítems, $\alpha=.82$ ) y Sobrecarga académica ( 3 ítems, $\alpha=.86$ ) con 5 opciones de respuesta tipo Likert (nunca, rara vez, algunas veces, frecuentemente y siempre) y que incluían la premisa al inicio de cada reactivo "Mi trabajo me causa estrés porque..."

Procedimiento Se solicitó inicialmente el permiso a las autoridades universitarias, se explicó los objetivos del estudio y ellas proporcionaron a los investigadores los correos institucionales de los profesores. Los correos se enviaron de manera personal explicando los objetivos de la investigación y señalando la liga de acceso al cuestionario.

La aplicación se realizó a través del software en línea Survey Monkey, que fue distribuido a 4000 profesores que contaba con correo institucional de un universo total de 5000 con una respuesta cercana al $10 \%$. Se solicitó la participación voluntaria de los profesores y se garantizó la confidencialidad de los datos proporcionados a través de la firma de consentimiento informado.

Se excluyeron del estudio los profesores que no completaron el cuestionario, también aquellos que en el mismo reportaron contar con un permiso que los eximía de estar frente a grupo, y aquellos que reportaron sufrir problemas de salud de origen no laboral.

Seguimiento: Las respuestas se integraron automáticamente a una base de datos en SPSS, que es una herramienta del Survey Monkey, se dio contestación a los correos de profesores que mostraron alguna duda al realizar el cuestionario. El primer corte se realizó dos semanas después del envío de la liga, y aunque la liga continuó abierta después de esta fecha ya no llegaron más cuestionarios.

Análisis Estadístico: Para el análisis estadístico se utilizó el paquete estadístico SPSS y AMOS, ambos en su versión 22, se utilizó análisis de frecuencias para establecer la prevalencia del SQT, y análisis de tendencia central, para establecer diferencias por grupo la prueba $t$ de muestras independientes y para las correlaciones la prueba producto momento de Pearson para asociar los FRPS-T y las dimensiones del SQT, y por último se construyó un modelo predictivo con el método de path analysis con los criterios de ajuste señalados por Ruiz, Pardo y San Martín (2010).

\section{Resultados}

Respecto a la prevalencia del SQT en esta población (ver Tabla 1), 11.7\% de profesores presenta muy baja o baja Ilusión por el trabajo, 20.7\% alto o crítico nivel de Desgaste psíquico, $10 \%$ presentan niveles altos o críticos de Indolencia, 17\% de Culpa y $17.6 \%$ niveles altos y críticos de SQT.

De acuerdo a los perfiles clínicos propuestos por Gil-Monte (2005) 8 docentes (2\%) presentan perfill de SQT (baja Ilusión, alto Desgaste, alta Indolencia) y $3(0.8 \%)$ presentan perfil 2 , la sintomatología señalada con Culpa. Los resultados de la prueba $t$ de muestras independientes (ver Tabla 2) señalan que las mujeres, los más jóvenes, los que tienen contrato de Asignatura, además, los que reciben estímulos presentan mayores niveles de Desgaste psíquico. Los jóvenes y solteros presentan mayores niveles de Indolencia y nuevamente los más jóvenes y con contrato de Asignatura, presentan mayores niveles de SQT. 
Tabla 1.- Prevalencia del SQT y sus dimensiones en profesores de Bachillerato

\begin{tabular}{|c|c|c|c|c|c|c|c|c|c|c|c|c|c|}
\hline & \multirow{2}{*}{ Media } & \multirow{2}{*}{ DT } & \multirow{2}{*}{$\begin{array}{c}\text { Intervalo } \\
\text { de } \\
\text { confianza } \\
95 \% \\
\end{array}$} & \multicolumn{2}{|c|}{$\begin{array}{l}\text { Muy } \\
\text { Bajo }\end{array}$} & \multicolumn{2}{|c|}{ Bajo } & \multicolumn{2}{|c|}{ Medio } & \multicolumn{2}{|c|}{ Alto } & \multicolumn{2}{|c|}{ Crítico } \\
\hline & & & & $\mathrm{F}$ & $\%$ & $\mathrm{~F}$ & $\%$ & $\mathrm{~F}$ & $\%$ & $\mathrm{~F}$ & $\%$ & $\mathrm{~F}$ & $\%$ \\
\hline Ilusión & 3.5 & 0.56 & $3.50-3.61$ & 13 & 3.3 & 33 & 8.4 & 177 & 44.8 & 172 & $43.5 \%$ & - & - \\
\hline Desgaste & 1.3 & 0.85 & $1.30-1.47$ & - & - & 170 & 43 & 43 & 36.2 & 57 & 14.4 & 25 & 6.3 \\
\hline Indolencia & 0.57 & 0.48 & $.527-.623$ & - & - & 166 & 42 & 190 & 48.1 & 23 & 5.8 & 16 & 4.1 \\
\hline Culpa & 0.57 & 0.54 & $.516-.623$ & - & - & 200 & 50.6 & 157 & 39.7 & 35 & 8.9 & 3 & 8 \\
\hline SQT15 & 0.74 & 0.46 & $.699-.791$ & - & - & 194 & 49.1 & 160 & 40.5 & 38 & 9.6 & 3 & 8 \\
\hline
\end{tabular}

Nota. DT= Desviación típica, F= frecuencia, SQT15= Incluye todas las dimensiones a excepción de Culpa

Tabla 2.- Resltados de la Prueba t de muestras independientes por grupo y dimensiones del $S Q T * p<.05, * * p<.001$

\begin{tabular}{lccccc}
\hline \multirow{2}{*}{ Sexo } & & Ilusión & Desgaste & Indolencia & Culpa \\
& Hombre & 3.5 & 1.2 & 0.58 & 0.62 \\
Edad & Mujer & 3.6 & $1.5^{*}$ & 0.56 & 0.51 \\
& $23-45$ & 3.5 & $1.5^{* *}$ & $0.63^{*}$ & 0.61 \\
\multirow{2}{*}{ Edo. Civil } & $46-70$ & 3.6 & 1.2 & 0.52 & 0.53 \\
& Sin pareja & 3.5 & 1.4 & $0.66^{*}$ & 0.61 \\
& Con pareja & 3.5 & 1.3 & 0.52 & 0.54 \\
& Asignatura & 3.5 & $1.4^{* *}$ & 0.6 & $0.6 *$ \\
& Carrera & 3.6 & 1.1 & 0.49 & 0.46 \\
Estímulos & (T.C.) & & & & \\
\hline
\end{tabular}

Nota: SQT 15= Incluye todas las dimensiones a excepción de Culpa

En cuanto a la prevalencia de FRPS-T observamos que los niveles más altos (30.9\%) son Estudiantes difíciles, seguido por la Mala relación con los compañeros (29.4\%) y Estresores físicos (26.1\%) (Ver Tabla 3). Las diferencias por sexo de acuerdo a los resultados de la prueba $t$ de student de muestras independientes, las mujeres presentan mayormente Malas relaciones con compañeros y Sobrecarga académica en comparación con los hombres. Los más jóvenes presentan mayor Sobrecarga académica y Falta de recursos en el Trabajo que los de mayor edad; los profesores de Asignatura presentan mayor de Falta de apoyo del jefe, Estudiantes difíciles, Inequidad y Falta de recursos para realizar su trabajo que los profesores de Carrera. Y por último los que reciben estímulos frente a los que no los reciben en la dimensión de Falta de apoyo del jefe (Ver Tabla 4). 
Tabla 3 .- Prevalencia de factores de riesgo psicosocial en el trabajo (FRPS-T) que son fuente de estrés en docentes de Bachillerato.

\begin{tabular}{|c|c|c|c|c|c|c|c|c|c|}
\hline \multirow{3}{*}{ FRPS-T } & \multirow{3}{*}{ Media } & \multicolumn{4}{|c|}{ Intervalo de } & \multirow{2}{*}{\multicolumn{2}{|c|}{ Medio }} & \multirow{2}{*}{\multicolumn{2}{|c|}{ Alto }} \\
\hline & & DT & Confianza & Bajo & & & & & \\
\hline & & & $95 \%$ & $\mathrm{~F}$ & $\%$ & $\mathrm{~F}$ & $\%$ & $\mathrm{~F}$ & $\%$ \\
\hline $\begin{array}{l}\text { Falta de recursos para realizar } \\
\text { su trabajo }\end{array}$ & 2.9 & 1.14 & $2.8-3.09$ & 168 & 42.5 & 128 & 32.4 & 99 & 25.1 \\
\hline Estudiantes difíciles & 2.5 & 0.78 & 2.4- 2.6 & 161 & 40.8 & 112 & 28.4 & 122 & 30.9 \\
\hline Falta de apoyo jefe & 2 & 0.86 & 1.9- 2.1 & 128 & 32.4 & 177 & 44.8 & 90 & 22.8 \\
\hline Mala relación con compañeros & 2.9 & 0.78 & $2.5-2.7$ & 126 & 31.9 & 153 & 38.7 & 116 & 29.4 \\
\hline Sobrecarga académica & 2.8 & 0.82 & $2.7-2.9$ & 134 & 33.9 & 276 & 44.6 & 85 & 21.5 \\
\hline Percepción de Inequidad & 2.9 & 0.91 & 2.8- 3.0 & 114 & 28.9 & 186 & 47.1 & 95 & 23 \\
\hline Estresores físicos & 3.2 & 0.85 & $3.1-3.3$ & 167 & 42.3 & 125 & 1.6 & 103 & 26.1 \\
\hline Percepción de Inseguridad & 2.3 & 0.82 & $2.2-2.4$ & 135 & 34.2 & 170 & 43 & 90 & 22.8 \\
\hline
\end{tabular}

Nota: FRPS-T $=$ Factores de riesgo psicocial en el trabajo, DT= Desviación Típica, F= frecuencia

Tabla 4.- Resultados de la Prueba t de muestras independientes por grupo y Factores de Riesgo Psicosocial.

\begin{tabular}{|c|c|c|c|c|c|c|c|c|c|}
\hline & & FAJ & MRC & $\mathrm{ED}$ & INE & IN & SA & $\mathrm{EF}$ & FR \\
\hline \multirow[t]{2}{*}{ Sexo } & Hombre & 1.9 & 2.5 & 2.5 & 2.8 & 2.3 & 2.7 & 3.2 & 2.9 \\
\hline & Mujer & 2 & $2.7^{*}$ & 2.5 & 3 & 2.3 & $2.9 *$ & 3.3 & 3 \\
\hline \multirow[t]{2}{*}{ Edad } & $23-45$ & 2.03 & 2.7 & 2.6 & 2.9 & 2.3 & $2.9 *$ & 3.3 & $3.3 * *$ \\
\hline & $46-70$ & 2.07 & 2.6 & 2.4 & 2.8 & 2.3 & 2.7 & 3.2 & 2.7 \\
\hline \multirow[t]{2}{*}{ Edo. Civil } & Sin pareja & 2.1 & 2.7 & 2.6 & 3 & 2.3 & 2.9 & 3.3 & 3.1 \\
\hline & Con pareja & 2 & 2.6 & 2.5 & 2.8 & 2.2 & 2.8 & 3.2 & 2.9 \\
\hline \multirow[t]{2}{*}{ Contrato } & Asignatura & $2^{*}$ & 2.6 & $2.6^{*}$ & $3^{*}$ & $2.3 *$ & 2.9 & 3.3 & $3.3^{* *}$ \\
\hline & Carrera (T.C.) & 1.8 & 2.5 & 2.4 & 2.6 & 2.1 & 2.7 & 3.1 & 1.7 \\
\hline \multirow[t]{2}{*}{ Estímulos } & $\mathrm{Si}$ & $2.08^{*}$ & 2.6 & 2.5 & 2.9 & 2.3 & 2.8 & 3.2 & 2.9 \\
\hline & No & 1.7 & 2.5 & 2.5 & 2.8 & 2.1 & 2.7 & 3.1 & 3.04 \\
\hline
\end{tabular}

Nota: $\mathrm{FAJ}=$ Falta de apoyo del Jefe, $\mathrm{MRC}=$ Mala relación con compañeros, $\mathrm{ED}=$

Estudiantes difíciles, INE = Inequidad. IN= Inseguridad, SA= Sobrecarga académica, $\mathrm{EF}=$ Estresores físicos, $\mathrm{FR}=$ Falta de recursos.

En la prueba de coeficiente de correlación producto momento de Pearson se encontró relación entre los FRPS-T y las dimensiones del SQT (ver Tabla 5), con puntuaciones que fluctuaron entre $\mathrm{r}=.16 \mathrm{y} \mathrm{r}=.54$. 
Tabla 5.- Correlación de dimensiones del SQT y FRPS-T en profesores de Bachillerato

\begin{tabular}{lcccccccc}
\hline Variable & INE & EF & FRT & SA & FAJ & ED & MRC & IN \\
\hline Ilusión & $-.22 * *$ & $-.20 * *$ & $-.25 * *$ & $-.18 * *$ & $-.25 * *$ & $-.18 * *$ & -.07 & $-.20 * *$ \\
Desgaste & $.35 * *$ & $.35 * *$ & $.37 * *$ & $.54 * *$ & $.31 * *$ & $.29 * *$ & $.32 * *$ & $.29 * *$ \\
Indolencia & $.33 * *$ & $.25 * *$ & $.31 * *$ & $.35 * *$ & $.31 * *$ & $.38 * *$ & $.25 * *$ & $.20 * *$ \\
Culpa & $.26 * *$ & $.22 * *$ & $.28 * *$ & $.22 * *$ & $.35 * *$ & $.37 * *$ & $.35 * *$ & $.16 *$ \\
\hline
\end{tabular}

Nota: $\mathbf{I N E}=$ Inequidad, $\mathbf{E F}=$ Estresores físicos, $\mathbf{F R T}=$ Falta de recursos para realizar su trabajo $\mathbf{S A}=$ Sobrecarga académica, $\mathbf{F A J}=$ Falta de apoyo del jefe, $\mathbf{E D}=$ Estudiantes difíciles, $\mathbf{M R C}=$ Mala relación con compañeros, $\mathbf{I N}=$ Percepción de inseguridad.

$p=*<0.05 * *<0.01$

La dimensión Ilusión correlacionó negativamente con casi todas las dimensiones de los FRPS-T a excepción de Malas relaciones con compañeros, como se esperaba, las correlaciones más altas se encontraron con Falta de Recursos para realizar su trabajo $(\mathrm{r}=-.25)$ y la Falta de Apoyo del jefe $(r=-.25)$. Las correlaciones entre Desgaste psíquico y FRPS-T fueron positivas resaltando Sobrecarga académica $(\mathrm{r}=.54)$ y Falta de recursos para realizar su trabajo $(\mathrm{r}=.37)$. En Indolencia también fueron positivas y más grandes con Estudiantes difíciles $(\mathrm{r}=.38)$ y Sobrecarga académica $(\mathrm{r}=.35)$. Por último, las correlaciones para Culpa también son positivas, y mayores en Estudiantes difíciles $(\mathrm{r}=.37)$ y Falta del Apoyo del jefe $(r=.35)$. Se realizó un modelo predictivo con los resultados obtenidos (Ver Figura 1) empleando el método de path analysis con los criterios de ajuste señalados por Ruiz, Pardo y San Martín (2010). Las dimensiones de Estresores Físicos e Inequidad no mostraron relevancia como predictores en las dimensiones del SQT, al proceder al ajuste, quedaron fuera. Las estimaciones hipotetizadas de los efectos directos entre todas las variables del modelo resultaron significativas y en la dirección esperada a excepción de Ilusión en el trabajo con Culpa.

Así, la Falta de recursos para realizar el trabajo $(\gamma=-.17, p<.05)$; Estudiantes difíciles $(\gamma=$ $-.15, p<.05)$; Falta de apoyo del jefe $(\gamma=-.16, p<.05)$ y Mala relación con compañeros $(\gamma=$ $-, 13, p<.05)$ se relacionaron negativa y significativamente con Ilusión en el trabajo.

La Sobrecarga académica $(\gamma=.45, p<.001)$; la Inequidad $(\gamma=.17, p<.001)$ y la Mala relación con los compañeros $(\gamma=.11 . p<.05)$ se relacionaron positiva y significativamente con Desgaste Psíquico. Estudiantes difíciles $(\gamma=.23, p<.001)$; Falta de Apoyo del Jefe $(\gamma=.13$, $p<.05)$ y Sobrecarga académica $(\gamma=.12, p<.05)$ se relacionaron positiva y significativamente con Indolencia. 
Con relación al proceso de SQT, la Ilusión por el trabajo obtuvo una relación negativa y significativa con Indolencia $(\beta=.24 p<.001)$ y positiva y no significativa con Culpa. Desgaste Psíquico obtuvo una relación positiva y significativa con Indolencia $(\beta=.17 p<.001)$ y con Culpa ( $\beta=.26 p<.001)$, al final para considerar el perfil tipo 2 la indolencia se relacionó significativa y positiva con Culpa $(\beta=.36 \mathrm{p}<.001)$.

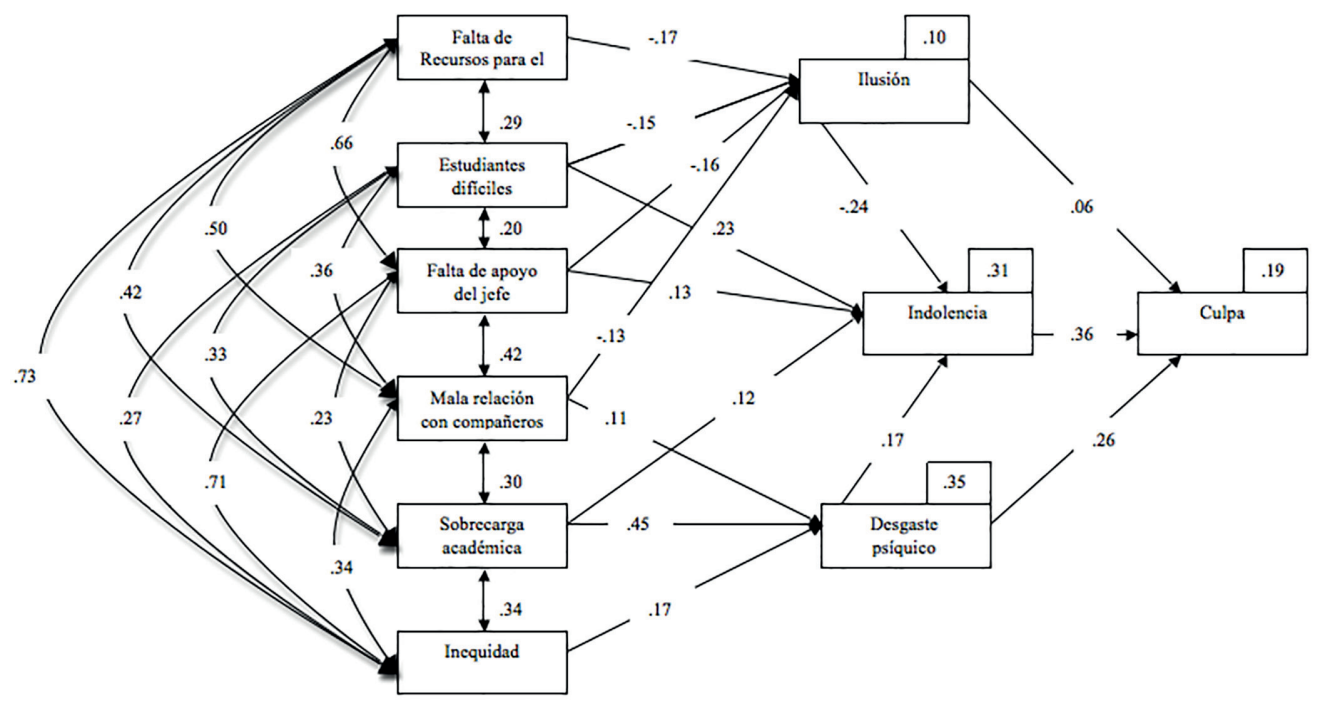

Figura 1. Modelo predictivo entre los FRPS-T y el modelo de SQT en profesores de Bachillerato

$\left(X^{2}=49.88, \mathrm{gl} .=1 X^{2} / \mathrm{gl} .=3 p<.05 ; \mathrm{IFI}=.976, \mathrm{TLI}=.930, \mathrm{CFI}=.976, \mathrm{GFI}=.976\right.$ y un $\mathrm{AGFI}=.916, \mathrm{RMSEA}=.073$.)

El modelo predictivo obtuvo un ajuste adecuado a los datos $\left(X^{2}=49.88, \mathrm{gl}=16, X^{2} / \mathrm{gl}=3\right.$, p <.001) acorde al tamaño de la muestra, los índices de adecuación del modelo presentados demuestran una buena adecuación $(\mathrm{IFI}=.976, \mathrm{TLI}=.930, \mathrm{CFI}=.976, \mathrm{GFI}=.976, \mathrm{AGFI}=$ .916 y RMSEA= .073). El Índice de Ajuste Incremental (IFI) presenta niveles por encima de .95 lo que indica que es adecuado; el TLI o Índice de ajuste no normado, permite ver la parsimonia del modelo en comparación con el nulo y muestra índices por encima de, 93 , lo que indica una buena parsimonia, el CFI es el Índice de ajuste comparado, presenta un ,97 lo que indica un buen ajuste con el modelo hipotetizado, el GFI que es un Índice de bondad de ajuste, presenta índices de .97 muy cercano a un ajuste perfecto, el AGFI que es un Índice ajustado de bondad de ajuste por los grados de libertad con el modelo nulo y presenta .91 , 
lo que refuerza el GFI y permite considerar aún más un buen ajuste, por último el Error de aproximación cuadrático medio (RMSEA) que mide la discrepancia por grado de libertad en términos de la población que presenta calificaciones por debajo del .08 (.073). Por lo anterior se concluye que el modelo presentó un adecuado ajuste global a los datos observados.

\section{Discusión}

El SQT es una de las afectaciones más frecuentes en los últimos tiempos dentro del colectivo docente, sobre todo por los impactos a la salud, las afectaciones en torno a la calidad educativa y a la formación del alumnado.

El objetivo del estudio fue establecer un modelo predictivo entre los factores de riesgo psicosocial propios del trabajo de docentes de bachillerato y la presencia del SQT. Los hallazgos del estudio inicialmente señalaron la prevalencia del síndrome en esta muestra de profesores que fue de $17.6 \%$ entre casos de SQT15 altos y críticos, en comparación con el 16.1\% reportado por Figueiredo-Ferraz, Gil-Monte y Grau-Alberola, (2009) en profesores portugueses y europeos incluyendo también casos altos y críticos. Pero por debajo de lo reportado por Marenco-Escuderos y Ávila-Toscano (2016) en una población de docentes de diferentes niveles educativos con un $20 \%$ y de profesores de educación básica de Montevideo que presentaron un total de $21.4 \%$ de casos con el síndrome (Ratto, García, Silva, González, 2015).

Otros estudios en México y en España con profesores de diversos niveles educativos reportan entre 4.5\%, 28.5\% y 77 \% (Diana, et al., 2014; Unda, García, Hernández, Esquivel y Tovalín, 2016; Villaverde, Unda, Escotto-Córdoba y Flores, 2019). Sin embargo, Reza, Plaza, Cuenca, López y Cruz (2019) señalan que los resultados de la prevalencia en docentes son muy dispares debido a la forma de medición y modelos utilizados.

Los niveles más altos de Factores de riesgo psicosocial en el trabajo que se presentaron fueron: Estudiantes difíciles, Mala relación con compañeros y Estresores físicos. Dos de estos factores también actuaron como predictores del síndrome. Esta posición es concordante con lo reportado por otros autores (Aloe, Amo y Shanahan, 2014; Caballero, 2018; López y Gil- Monte, 2015).

De acuerdo a los resultados, el modelo mostró como la Ilusión fue predicha por la combinación de varios FRPS-T; la Falta de recursos para el trabajo, Estudiantes difíciles, Falta de apoyo del jefe y Mala relación con compañeros que presentó una relación directa y negativa; la motivación en el trabajo y el clima laboral se encontraron directamente relacionados con la presencia de la Ilusión por el trabajo. Saltijeral y Ramos (2015) señalan que en escuelas de la Ciudad de México de educación secundaria, se reportaron situaciones de violencia hacia los profesores, como amenazas, robos y asaltos por parte de sus estudiantes y ante esta situación, recibieron poco o nulo apoyo de sus jefes, por otro lado, es común escuchar en las escuelas quejas de profesores que señalan estar demandados por padres de familia por considerar alguna mala actuación de su parte hacia los alumnos.

Por lo tanto, es necesario señalar que la actividad medular del maestro es la relación directa con sus alumnos y al verse afectada por razones de rechazo, violencia o falta derespeto 
puede repercutir directamente en la percepción de autoeficacia, autoestima y seguridad en el desempeño y logros de su trabajo, y ser una fuente impórtate de desvalorización de sí mismo como profesional provocando el incremento de estrés (Cardozo, 2016).

Pineda y García (2016), plantean que las relaciones entre alumnos y profesores pueden tornarse conflictivas y complejas debido al choque de generaciones y culturas además de la animadversión y el desinterés de los alumnos por los conocimientos propuestos. Aunado a lo anterior los ambientes de trabajo en las escuelas se han tornado difíciles entre los mismos profesores lo que incrementa los conflictos personales (López y Gil-Monte, 2015); consideramos que en México, también son producto del incremento de una fuerte competencia para conservar sus empleos, lo que contribuye a destruir el tejido social y propicia un pobre apoyo social.

Es necesario señalar que más del 50\% de profesores de la muestra tiene contrato temporal y salarios precarios lo que puede contribuir a explicar estos factores de riesgo asociados a la Ilusión. Caballero (2018) señala que el sentirse con poco reconocimiento o apoyo los profesores se ven directamente afectados en su autoeficacia y motivación, Marenco-Escudero y Ávila-Toscano (2016) señalan que la variable apoyo social en los docentes está altamente relacionada con solventar eventos generadores de estrés pues quien lo proporciona ofrece recursos emocionales, materiales e informativos, por el contrario la disminución o carencia de apoyo tiene relación con la presencia de desgaste y agotamiento emocional además de baja realización personal en el trabajo.

Al respecto Colino y Pérez de León, (2015), señalan que la percepción de injusticia en el trabajo y de un mal ambiente social están asociados con el SQT ya que generan frustración y desánimo lo que es el factor más destructivo.

Cabe señalar que a su vez la Ilusión por el trabajo tuvo un relación directa y negativa con la Indolencia y directa con la Culpa, pero no fue significativa, lo que concuerda con lo reportado en el estudio con profesores de secundaria de Rabasa, Figueiredo-Ferraz, Gil-Monte, y Llorca-Pellicer (2016)

Siguiendo con el proceso, la Sobrecarga académica, la Inequidad en el trabajo y la Mala relación con compañeros predijo de forma directa positiva y significativa al Desgaste psíquico. En diversos estudios se ha asociado la Sobrecarga laboral a un ambiente organizacional inadecuado como predictor del Desgaste psíquico, (López y Gil-Monte, 2015; Rabasa, Figueiredo-Ferraz, Gil-Monte, y Llorca-Pellicer, 2016; Souto, Pereira, Brito, Sancho y Jardim, 2018). La Reforma educativa del 2013 en México incremento e incorporó nuevas exigencias al trabajo de los profesores como: jornadas de trabajo más largas e intensas, diversificación de actividades fuera del aula y en contraparte las condiciones laborales fueron precarizandose a través de bajos salarios, contrataciónes temporales, mayor supervisión a través de programas de evaluación al desempeño, evaluación a la productividad, deshomologación salarial, entre otros, lo que provocó ambientes poco solidarios, competitivos y contribuyó a percibir más Inequidad derivando en un incremento de conflictos entre los profesores y los jefes, creando un ambiente dificil que minimiza el apoyo social (Palacios, Morales y Estrella, 2018; Unda, 2018). 
El Desgate psíquico a su vez, en el modelo, presenta una relación directa y positiva con Indolencia y con la Culpa como lo señala el proceso del SQT (Gil-Monte, 2005) aunque en menor medida con Indolencia.

El modelo ubica a la Indolencia como resultado de la pérdida de la Ilusión por el trabajo y el Desgaste psíquico, pero los reultados del modelo señalan también como predecesores de la misma a FRPS-T como la Falta de apoyo del jefe, la Sobrecarga académica y los Estudiantes difíciles, Gil-Monte (2005) señala que la Indolencia es una variable actitudinal del síndrome y que funciona como una forma de afrontamiento ante situaciones donde el profesor se ve rebasado, estas actitudes cínicas, frias y distantes permiten al docente continuar con su trabajo, aún a costa de tener comportamientos disfuncionales en su proceder profesional.

Es necesario señalar que algunos profesores que se sienten incómodos con éste proceder, pueden sentirse avergonzados y manifestar sentimientos de culpabilidad y decidir cambiar sus actitudes apelando a su vocación, como forma para reivindicar el compromiso por su trabajo y realizar nuevos esfuerzos cognitivos y emocionales por mejorar profesionalmente. Lo que a la larga repite el proceso hasta llevar a un daño mayor y afectar tanto la salud de los profesores como a la propia institución educativa. El modelo que se obtuvo muestra como la Indolencia es un precursor importante de la Culpa (.36) lo anterior permite reflexionar en la necesidad de incorporar en el proceso del SQT a la Culpa para explicar mejor los daños en los profesores que padecen SQT (Perfil 2) (Rabasa, Figueiredo-Ferraz, Gil-Monte, y Llorca-Pellicer, 2016). Al respecto Fiorilli, Albanese, Gabola y Pepe (2017) señalan en su estudio que los docentes con SQT tienden a tener más riesgo de percibir las emociones negativas de manera más intensa durante un conflicto verbal con sus alumnos que los profesores sin SQT, lo que redunda en menor efectividad en su trabajo diario, baja autoestima e insatisfacción laboral.

En conclusión los resultados indican que el SQT en profesores de Bachillerato se puede predecir por los FRPS-T como Sobrecarga académica, Estudiantes difíciles, Inequidad en el trabajo, Falta de recursos para el trabajo, Falta de apoyo del jefe y Mala relación con compañeros Varios autores mencionan estos factores de riesgo laboral en población de docentes (Buonomo, Fatigante y Fiorilli, 2017).

Es necesario hacer la aclaración que los resultados obtenidos pueden deberse a la composición de la muestra, ya que la mayor parte son profesores de Asignatura (76.5\%) cuya función contractual es impartir horas frente a grupo, aunque realicen actividades extras sin pago, como asesorar estudiantes en actividades extracurriculares, planeación, evaluación, elaboración de materiales didácticos, participación en grupos de trabajo, elaboración de libros de texto, asesorías a alumnos irregulares, cursos sabatinos, entre otras, y por lo tanto son quienes tienen un mayor trato con la población estudiantil, así como un elevado número de alumnos y de horas de trabajo, las cuales obligan al docente a llevar un ritmo muy apurado y sin descansos. Como consecuencia puede causar la sensación de estar excesivamente sobrepasados, con presión constante por el tiempo y se encuentran menos orientados a la tarea (Ghanizadeh y Ghonsooly, 2014). Esta condición es además propicia para sufrir Desgaste psíquico e Indolencia que es traducida en sentimientos negativos y actitudes de 
cinismo en contra de los alumnos además de limitar de forma considerable la labor docente (Díaz y Gómez, 2016).

Por otro, lado aunque el estudio de Spilt, Koomen y Thijs (2011) asocia los problemas de mala conducta e indisciplina del estudiante a la aparición del Desgaste psíquico, nuestros resultados señalan que los Estudiantes difíciles predicen la baja Ilusión y la Indolencia incrementando el proceso de gravedad del SQT y de la aparición de la Culpa en el docente, esto puede indicar que la relación entre alumno-docente cobra mayor relevancia en esta población en los profesores de asignatura ya que tienen más grupos a cargo y mayor estudiantes que los profesores a tiempo completo. En este bachillerato los profesores de tiempo completo reducen las horas frente a grupo e incrementan actividades de investigación y de divulgación. Estas particularidades en los docentes de bachillerato son similares a otros estudios que reportan en docentes mexicanos que imparten en el mismo nivel (Amador, Rodríguez, Serrano, Olvera y Martínez 2014).

La Sobrecarga académica tuvo el mayor porcentaje de predicción (45\%) en relación a la dimensión de Desgaste psíquico, y la baja Ilusión en el trabajo un porcentaje predictivo mayor que el Desgaste psíquico para predecir la Indolencia.

\section{Aportes}

El modelo predictivo propuesto en esta investigación pone de manifiesto que la organización, el contenido del trabajo y la realización de la tarea se encuentran relacionadas con la aparición de síndrome.

Los resultados del modelo, permiten caracterizar los Factores de riesgo psicosocial propios de la actividad docente de profesores de Bachillerato de la Ciudad de México asociados al SQT y sus dimensiones, que pueden ser guía y referente de programas preventivos de estrés y SQT dentro de la población de docentes.

Los resultados también confirman el proceso de desarrollo del SQT propuesto por Gil-Monte (2005) y que ha sido probado en otros estudios como el de Rabasa, Figueiredo-Ferraz, Gil-Monte, y Llorca-Pellicer, (2016), pues tienen gran coincidencia.

\section{Limitaciones del Estudio}

La tasa de respuesta de éste estudio fue baja (10\%) y a diferencia de la mayoría de investigación que se realiza en México, fue de manera digital a través de un programa llamado Survey Monkey por medio del envío del cuestionario a través de una liga en el correo electrónico institucional. Queda la duda si los profesores que contestaron el cuestionario fue porque tenían mayor tiempo para hacerlo, lo que puede sesgar los resultados y considerar que el problema de presencia del SQT en esta población fue mayor, o, por el contrario, los profesores que se animaron a contestarlo fue porque consideraban importante responder para evidenciar las condiciones en las que se encuentran y la población muestra sea la más afectada. Por lo tanto, es necesario seguir con nuevas investigaciones y con muestras representativas y aleatorias en esta población de profesores para confirmar los hallazgos y ampliar las posibilidades de incluir estudios de corte longitudinal. 
Otra limitación del estudio y que señala áreas de oportunidad de acuerdo a los hallazgos, es analizar de manera mas profunda el papel que juega las diferncias por sexo, por tipo de nombramiento (profesores de Asignatura y de Carrera), tipo de contrato, (profesores temporales o definitivos). Esto permitirá observar diferencias mas puntuales de los FRPS-T para cada grupo y su contribución para analizar el SQT y su prevención.

Otra limitación fue no integrar al modelo las posibles afectaciones tanto organizacionales (absentismo) e individuales como de síntomas de salud mental (depresión, ansiedad). Por lo que se sugiere para nuevas investigaciones. Y tener un modelo integral de antecedentes y consecuentes del SQT en profesores de Bachillerato.

También, sería conveniente continuar probando el modelo de FRPS-T y su relación con el SQT en profesores de diferentes niveles educativos.

\section{Implicaciones Prácticas}

La contribución del estudio fue demostrar como el conjunto de FRPS-T que incluimos actúan como estresores laborales en las organizaciones educativas y son precursores del SQT y sus dimensiones, mermando la calidad de vida del trabajador docente y su salud. De lo anterior se derivan las siguientes recomendaciones

Trabajar en las organizaciones educativas con información al respecto del SQT y cuales son las condiciones de su trabajo que lo propician; además es necesario en la distribución de tareas, cuidar el no sobrecargar al docente de actividades adicionales a la atención de los estudiantes, promover espacios de convivencia que mejoren las relaciones entre compañeros para ampliar el apoyo social y hacer frente al estrés. También incluir códigos de convivencia para mejorar la relación entre estudiantes y profesores que permitan un desarrollo más armónico en el proceso educativo y evitar actos de violencia, además de promover valores de respeto y reconocimiento del trabajo docente. Otro elemento importante de aporte es la necesidad de formación de los jefes, pues en el ámbito educativo son los mismos docentes que son asignados a puestos académico-administrativos sin capacitación para ejercer funciones adecuadas de liderazgo. Tener presente que la actividad desarrollada por los docentes es central, no solo para los estudiantes en formación, sino para la sociedad en su conjunto,

Es necesario que las instituciones cuenten con programas de prevención del estrés y mejoren las condiciones de trabajo de los profesores para evitar mayores daños tanto a la salud como a las organizaciones educativas.

Los hallazgos contribuyen también a considerar el modelo de cuatro dimensiones del SQT como un elemento valioso para explicar el proceso, pero también para las aplicaciones prácticas al reconocer los dos perfiles señalados e identificar y diferenciar los daños.

\section{Referencias}

Aloe, A. M., Amo, L. C., y Shanahan, M. E. (2014). Classroom Management Self-Efficacy and Burnout: A Multivariate Meta-Analysis. Educational Psychology Review, (26), 101-126. http://dx.doi.org/10.1007/s 10648-013-9244-0 
Amador, R., Rodríguez, C., Serrano, J, Olvera, J. Martínez, S. (2014) Estrés y Burnout en docentes de educación media superior. Medicina Salud y Sociedad, 4(2), 119-141. http://www.medicinasaludysociedad.com.

Blanch, J. (2008). La doble cara organizacional y personal de la flexibilización del trabajo. Desafíos, responsabilidad y agenda de la Psicología Social del Trabajo y de la organización. Perspectivas en psicología, (11), 11-26. https://www.umanizales.edu.co/publicaciones/ campos/sociales/perspectivas psicologia/html/revistas contenido/revista11/Ladoblecara.pdf

Buonomo, I., Fatigante, M., y Fiorilli, C. (2017). Teachers' burnout profile: Risk and protective factors. The Open Psychology Journal, 1011) 190-201 DOI: 10.2174/1874350101710010190

Caballero, F., (2018). Afectación del síndrome de estar quemado en el trabajo o burnout. Medidas preventivas y de intervención actuales. Revista de Innovación Didáctica de Madrid. (52.), 39-82 https://www.csif.es/contenido/comunidad-de-madrid/ensenanza/205631

Cardozo L. (2016). El estrés en el profesorado. Revista de Investigación Psicológica, (15), 75-98. http://www.scielo.org.bo/scielo. php? script $=$ sci arttext\&pid $=$ S2223-30322016000100006\&lng $=$ es\&tlng $=$ es

Colino, N., Pérez de León, P., (2015) El síndrome de Burnout en un grupo de profesores de enseñanza secundaria en Montevideo Ciencias Psicológicas, 9(1), 27-41

Diana, I., Hernández, M., Fiz, I., López, A., González, C., y Pariente, A., (2014). Síndrome de burnout en docentes de infantil, primaria, secundaria, formación profesional y bachillerato de Albacete, Cuenca y Toledo. Seguridad y Salud en el Trabajo, (80), 18-29 https:|| www.fundacionmapfre.org/documentacion/publico/i18n/catalogo imagenes/imagen id.cmd?idlmagen = 1093204

Díaz, F., Gómez, I. (2016) La investigación sobre el síndrome de burnout en Latinoamérica entre 2000 y el 2010. Psicología desde el caribe. (33), 113-131 http://dx.doi.org/10.14482/psdc.33.1.8065

Ducoing, P., (2018), Educación básica y reforma educativa. México, Universidad Nacional Autónoma de México. http:/|132.248.192.241/ editorial/wp-content/uploads/2018/05/Educacio\%CC\%81n-ba\%CC\%81 sica-y-reforma-educativa.pdf

Fernández-Puig, V., Longás. Mayayo, J., Chamarro Lusarby, A. y Virgili Tejedor, C. (2015). Evaluando la salud laboral de los docentes de centros concertados: el Cuestionario de Salud Docente. Journal of Work and Organizational Psychology, (30), 1-11.

Fernet, C., Guay, F., Senécal, C., y Austin, S. (2012). Predicting intraindividual changes in teacher burnout: The role of perceived school environment and motivational factors. Teaching and Teacher Education, 28(4), 514-525. doi:10.1016/j.tate.2011.11.013

Figueiredo-Ferraz, H., Gil-Monte, P., y Grau-Alberola, E. (2009). Prevalencia del síndrome de quemarse por el trabajo (Burnout) en una muestra de maestros portugueses. Aletheia, 29, 6-15. Doi:10.1590/S0034-89102008000300009

Fiorilli C, Albanese 0, Gabola y P, Pepe A (2017) Teachers' emotional competence and social support: Assessing the mediating role of teacher burnout. Scandinavian Journal of Educational Research, 61(2), 127-38. http://dx.doi.org/10.1080/00313831.2015.1119722

García-Carmona, M., Marín, M. D., y Aguayo, R. (2018), Burnout syndrome in secondary school teachers: A systematic review and metaanalysis. Social Psychology of Education, 22(1), 189-208. https://doi.org/10.1007/s 11218-018-9471-9

Ghanizadeh, A., y Ghonsooly, B. (2014). A tripartite model of EFL teacher attributions, burnout, and self-regulation: Toward the prospects of effective teaching. Educational Research for Policy and Practice, 13, 145-166. Doi:10.1007/s10671-013-9155-3

Gil- Monte, (2005), El síndrome de Quemarse por el trabajo, (Burnout): Una enfermedad laboral en la sociedad del bienestar, Madrid: Pirámide

Gil-Monte, P., Unda, S., y Sandoval, O. (2009). Validez factorial del Cuestionario para la Evaluación del Síndrome de Quemarse por el Trabajo (CESQT). Salud Mental, 32(3), 205-2014. http://www.psiquiatria.com/imprimir.ats?24872

Gil-Monte, P., Viottib, S., Converso, D., (2017), Propiedades psicométricas del Cuestionario para la Evaluación del Síndrome de Quemarse por el Trabajo (CESOT) en profesionales de la salud italianos: una perspectiva de género, Liberabit, 23(2), 153-168, https://doi. org/10.24265/liberabit.2017.v23n2.01

Juárez-García, A., Idrovo, Á., Camacho-Ávila, A., y Placencia-Reyes, 0. (2014). Síndrome de burnout en población mexicana: Una revisión sistemática. Salud Mental, 37(2), 159-176. http://www.medigraphic.com/pdfs/salmen/sam-2014/sam142j.pdf

Maslach, C. (2003). Job Burnout: New Directions in Research and Intervention. Current Directions in Psychological Science, 12, $189-192$. doi: $10.1111 / 1467-8721.01258$

Marenco-Escuderos, A., y Ávila-Toscano, J. (2016). Dimensiones de apoyo social asociadas al síndrome de burnout en docentes de media académica. Pensamiento Psicológico, 14(2), 7-18 https://revistas.javerianacali.edu.co/index.php/pensamientopsicologico/article/ view/1237

Olivares, V., y Gil-Monte, P. (2009). Análisis de las Principales Fortalezas y Debilidades del "Maslach Burnout Inventory" (MBI). Ciencia y Trabajo, 33, 160-167. 
Palacios, R., Morales, E. y Estrella, G. (2018). Sobrecarga laboral, tensiones dentro y fuera del aula: docentes enfermos, estresados. Intercambio, (12), 17-21. http://idea-network.ca/wp-content/uploads/2018/04/4-Sobrecarga-laboral.pdf

Pineda, J, y García, F., (2016). Conflicto y convivencia: profesores y alumnos en el proceso de enseñanza en un aula de secundaria. Revista mexicana de investigación educativa, 21(71), 1073-1091. http://www.scielo.org.mx/scielo.php?script=sci arttext\&pid=\$1405. $66662016000401073 \&$ lng $=$ es\&tlng $=$ es.

Rabasa, B., Figueiredo-Ferraz, H., Gil-Monte, P. y Llorca-Pellicer, M., (2016) El papel de la Culpa en la relación entre el síndrome de quemarse por el trabajo y la inclinación al absentismo de profesores de Enseñanza Secundaria. Revista de Psicodidáctica, 21(1), 103-119 http://www.ehu.eus/0js/index.php/psicodidactica/article/viewFile/13076/13429

Ratto D. García R., Silva, M., y González, M., (2015). El síndrome de quemarse por el trabajo y factores psicosociales en docentes de primaria de la ciudad de Montevideo. Ciencias Psicológicas, 92) ,273-281. http://www.scielo.edu.uy/scielo.php?script=sci arttext\&pid $=$ S1688-2212015000300005\&lng = es\&tlng = es

Reza, L., Plaza, C., Cuenca, C., López, A., Cruz, G., (2019), El síndrome de Burnout y su repercusión en el rendimiento académico, Revista Atlante: Cuadernos de Educación y Desarrollo, 1-29 https://www.eumed.net/rev/atlante/2019/02/index.html

Ruiz, M., Pardo, A., y San Martín, R. (2010). Modelos de ecuaciones estructurales. Papeles Del Psicólogo, 31(1), 34-45.

Saltijeral, M. y Ramos, L. (2015). Identificación de estresores laborales y burnout en docentes de una secundaria para trabajadores del Distrito Federal. Salud Mental, 38(5), 361-369.

Sánchez de Gallardo, M. y Mantilla de Gil, M. (2005). Situaciones generadoras de los niveles de estrés en docentes universitarios. Revista Venezolana de Ciencias Sociales, 9(1) 140-162.

Serrano Díaz, N. y Guil Bozal, R. (2014). Características sociodemográficas y Síndrome de Burnout en el profesorado de Educación Infantil de la Bahía de Cádiz. Journal for Educators, Teachers and Trainers, 5(2), 226-240.

Spilt, J., Koomen, H., y Thijs, J. (2011). Scandinavian Journal of Educational Research Teacher Wellbeing: The Importance of Teacher-Student Relationships. Educational Psychology Review, 23, 457-477. Doi:10.1007/s 10648-011-9170-y

Souto, I., Pereira, A., Brito, E., Sancho, L., y Jardim, J. (2018). Psychosocial Risk Factors and Distress in Higher Education Teachers. The European Proceedings of Social \& Behavioural Sciences, 127-140 https://www.futureacademy.org.uk/files/images/upload/ icH\&Hpsy2018F14.pdf

Unda, S. (2015) Factores de Riesgo Psicosocial laboral asociados a la aparición del Sindrome de Quemarse por el Trabajo (Burnout) y sus consecuencias en la salud en profesores universitarios. (Tesis doctoral) Universidad Nacional Autónoma de México, México.

Unda, S. (2018). Condiciones de trabajo y salud, un rubro pendiente en la defensa de los derechos de los docentes. Intercambio, (12), 22-25.

Unda, S., Uribe, F., Jurado, S., García, M., Tovalín, H., y Juárez, A. (2016). Elaboración de una escala para valorar los factores de riesgo psicosocial en el trabajo de profesores universitarios. Revista de Psicología del Trabajo y las Organizaciones, 32(2) 67-74. http:// doi.org/10.1016/j.rpt0.2016.04.004

Unda Rojas, S, García Arreola 0, Hernández Toledano, A, Esquivel Lozada C, Tovalin Ahumada, H. (2016) Enfermedades en personal educativo y su asociación con el Síndrome de Quemarse por el Trabajo en docentes. Revísta Mexicana de Salud en el Trabajo. 7-8(17-18): 23-30.

Villaverde, D., Unda, S., Escotto, E., y Flores R. (2019). Rasgos de personalidad predictores del Síndrome de Quemarse por el Trabajo en profesores mexicanos. Propósitos y Representaciones, 73). doi: http://dx.doi.org/10.20511/pyr2019.v7n3.346 